\title{
ANÁLISE DO IMPACTO DA PRODUÇÃO FLORESTAL NO DESENVOLVIMENTO ECONÔMICO E AMBIENTAL DE MATO GROSSO DO SUL
}

\author{
Alexander Bruno Pegorare ${ }^{1}$, Dany Rafael Fonseca Mendes², Paula Martin de Morais ${ }^{3}$, Reginaldo Brito \\ da Costa ${ }^{4}$, Michel Constantino ${ }^{5}$
}

\begin{abstract}
RESUMO - O Brasil é o maior exportador de celulose do mundo, e a maior expansão do setor florestal brasileiro ocorreu em Mato Grosso do Sul, especialmente com relação à produção de celulose e papel. O desenvolvimento gerado pelo setor madeireiro, de florestas nativas e plantadas, pode ser visto como uma combinação de fatores internos e externos, com aplicação na cadeia produtiva. Nesse contexto, a presente pesquisa teve por objetivo avaliar o impacto da produção de produtos madeireiros de florestas nativas e plantadas no desenvolvimento econômico e ambiental do Estado de Mato Grosso do Sul, região Centro-Oeste do Brasil, entre 2000 e 2014. O procedimento metodológico consistiu em pesquisa exploratória sobre a atividade setorial, com o uso de banco de dados secundários disponíveis nos sites do Instituto Brasileiro de Geografia e Estatística (IBGE) para extração das variáveis econômicas e ambientais, aplicando-se modelos econométricos de regressão múltipla com dados longitudinais. Os resultados mostraram externalidades positivas geradas pela produção de madeira plantada para o desenvolvimento econômico e, ainda, para o meio ambiente. A produção madeireira de florestas nativas não apresentou impacto econômico, influenciando negativamente o meio ambiente. O impacto gerado pela liberdade econômica também foi analisado, ou seja, o grau de abertura comercial no setor, resultando choque positivo da política de atração de investimentos para o setor agroflorestal. As evidências empíricas e os modelos econométricos apresentados podem ser utilizados para auxiliar os policy-makers e produtores na tomada de decisões mais coerentes e sistêmicas, contribuindo para o setor no qual o Brasil é maior exportador mundial. A pesquisa ainda contribui para uma lacuna na literatura, com estudos aplicados para as atividades produtivas da região Centro-Oeste do Brasil.
\end{abstract}

Palavras chave: bioeconomia, econometria, florestas nativas e plantadas, modelagem, setor agroflorestal.

\section{ANALYSIS OF THE IMPACT OF FOREST PRODUCTION ON THE ECONOMIC AND ENVIRONMENTAL DEVELOPMENT OF MATO GROSSO DO SUL}

\begin{abstract}
Brazil is the largest cellulose pulp exporter in the world, and the largest expansion of the Brazilian forestry sector occurred in Mato Grosso do Sul, especially with production of pulp and paper. The development generated by the timber sector, of native and planted forests, can be seen as a combination of internal and external factors, with application in the production chain. In this context, this research aimed to evaluate the impact of the production of timber products from native forests and planted in the economic and environmental development of the state of Mato Grosso do Sul, Midwest region of Brazil, between 2000 and 2014. The methodological procedure consisted of exploratory research on the sectorial activity, with the use of secondary databases available on the sites of the Brazilian Institute of Geography and Statistics (IBGE) for the extraction of economic variables and by applying multiple regression econometric models with longitudinal data. The results showed positive externalities generated by the production of timber planted for economic development and also for the environment. The timber production of native forests had no economic impact, negatively influencing the environment. The impact generated by economic freedom was also analyzed, that is, the degree of commercial
\end{abstract}

\footnotetext{
${ }^{1}$ IBGE - Instituto Brasileiro de Geografia e Estatística - E-mail: alexander.pegorare@ibge.gov.br;

${ }^{2}$ UniCEUB - Centro Universitário de Brasília - E-mail: rafael.dany@gmail.com;

${ }^{3}$ UCDB - Universidade Católica Dom Bosco - E-mail: paulamartin.bio@gmail.com;

${ }^{4}$ UCDB - Universidade Católica Dom Bosco - E-mail: reg.brito.costa@gmail.com;

${ }^{5}$ UCDB - Universidade Católica Dom Bosco - E-mail: michel@ucdb.br.
} 
openness in the sector, resulting in a positive shock of the policy of attracting investments to the agroforestry sector. The empirical evidences and econometric models presented can be used to help the policy-makers and producers in making more coherent and systemic decisions, contributing to the sector in which Brazil is the world's largest exporter. The research still contributes to a gap in the literature, with studies applied to the productive activities of the Midwest region of Brazil.

Keywords: bioeconomics, econometrics, native and planted forests, modeling, agroforestry sector.

\section{INTRODUÇÃO}

Nas últimas décadas, estudos sobre impactos gerados pela atividade produtiva e sua relação com o meio ambiente foram exponencialmente impulsionados. Com a saída dos Estados Unidos da América do Acordo de Paris, esta pauta de análise voltou ao foco da discussão em todos os setores da economia, nomeadamente na área do desenvolvimento (Herrera et al., 2018).

As externalidades (positivas e negativas) geradas pelas atividades produtivas do meio rural são acompanhadas de pesquisas e debates relacionados com o meio ambiente. É, portanto, preciso integrar práticas sustentáveis de dimensão social e ambiental a fim de garantir sua perenidade econômica. Atualmente, há uma grande preocupação mundial com a degradação ambiental originada das atividades econômicas exploratórias de meios naturais, especialmente pela mudança no uso da terra. Segundo a FAO/IPCC (2017), esta alteração de uso se refere às mudanças provocadas na utilização da terra por ações antrópicas (como fontes de emissão ou remoção de gases que causam o efeito estufa), as quais podem acarretar alterações do sistema climático local, regional e, até mesmo, global.

Explicitada a necessidade da ciência econômica "teorizar" sobre a interação do sistema econômico com o meio ambiente, duas hipóteses surgem na pesquisa da economia do meio ambiente: $i$ ) se a mudança do uso da terra ocorrer de forma equivocada, isto poderia ocasionar problemas econômicos ambientalmente insustentáveis; e, ii) se utilizados de forma acertada, esses recursos poderiam ser a chave do desenvolvimento econômico e da promoção de bem-estar social.

Entre as atividades de fins econômicos relacionadas ao setor agroflorestal, duas se encontram equidistantes na utilização da área, porém capazes de gerar receitas e impactar a economia: a atividade de supressão vegetal, utilizada para a exploração de florestas nativas; e as áreas reflorestadas com fins comerciais.
Buscando abordar o efeito conjunto da produção das florestas nativas e plantadas no âmbito econômico ambiental, o objetivo deste trabalho foi avaliar o impacto da produção bruta das florestas nativas e plantadas (utilizadas para produção de madeira, lenha, carvão e celulose) sobre o crescimento econômico no Estado de Mato Grosso do Sul (Brasil) entre 2000 e 2014. O foco da pesquisa foi avaliar se o grau de abertura comercial foi relevante para a produção madeireira florestal, bem como se este crescimento econômico, através das emissões ou remoções de gases causadores do efeito estufa pela atividade agropecuária, impacta na degradação ambiental.

A pesquisa é baseada em modelos econométricos, que buscam mensurar a relação linear entre a economia verde emergente da extração de produtos madeireiros de florestas nativas e plantadas com o desenvolvimento econômico do Estado, medido pelo Produto Interno Bruto (PIB) e Produto Interno Bruto per capita (PIBpc), e, no meio ambiente, analisando o impacto gerado nas emissões de $\mathrm{CO}_{2}$.

O estudo avalia empiricamente o Estado com maior produção de celulose do Brasil, que é o segundo país em produção mundial (IBA, 2017), aplicando modelagem ainda não utilizada na literatura, pois compõe análise de modelos econômico e ambiental, com propósito de entender diferentes impactos causados pelo desenvolvimento do setor agroflorestal. A análise pode contribuir para tomada de decisão de policy-makers, gestores, produtores rurais e especialistas da área.

Para o alcance do objetivo, este manuscrito foi dividido nesta introdução, na próxima seção (que trata do panorama do desenvolvimento do Mato Grosso do Sul e os principais estudos de impacto), na seção 3 (na qual foram delimitados os materiais e procedimentos metodológicos), seguida dos resultados (seção 4) e considerações finais ( seção 5). 


\section{DESENVOLVIMENTO ECONÔMICO, MEIO AMBIENTE E SETOR AGROFLORESTAL NO MATO GROSSO DO SUL - REGIÃO CENTRO-OESTE}

No âmbito das políticas públicas, pode-se extrair a ideia de que o desenvolvimento rural deve combinar o aspecto econômico (como o aumento do nível e da estabilidade da renda familiar) com o aspecto social (obtenção de uma condição de vida socialmente aceitável), além de condições ambientalmente sustentáveis (Dalchiavon \& De Mello, 2015). Dentro desse contexto, o setor econômico de Mato Grosso do Sul passou por uma reorganização produtiva, inserindo-se num processo de modernização, melhorando a competitividade e consolidando uma estrutura de produção adaptada para a concorrência. Em 2014, o Produto Interno Bruto do estado foi de $\mathrm{R} \$ 78,9$ bilhões, representando 1,2\% da participação no PIB nacional daquele ano, com crescimento real acumulado de $530 \%$ no período de 2000 a 2014. Em 2017, o Mato Grosso do Sul foi o segundo estado a ter melhor desempenho do PIB, atrás apenas de Mato Grosso (IBGE, 2017).

O Estado de Mato Grosso do Sul se destacou nacionalmente ao apresentar resultados expressivos na agropecuária. De acordo com o Instituto Brasileiro de Geografia e Estatística (2017), a participação da atividade primária (agropecuária) no PIB sul-matogrossense foi de $15,45 \%$ em 2014. Nesse panorama, considerando o comércio exterior, o estado contribuiu com $1,51 \%$ do total das exportações do Brasil, ou seja, US\$ 5,2 bilhões (MDIC, 2017). Com relação às importações, a participação do Mato Grosso do Sul foi de 1,81\%, aproximadamente US\$ 5,7 bilhões (MDIC, 2017).

Em função da necessidade de aliar crescimento econômico sustentável aplicado às políticas de investimento, as questões ambientais devem ser adequadas e devem ser levadas em consideração durante as tomadas de decisão. Isso porque a degradação ambiental é causada por fatores-chave, como industrialização, transporte, população, pobreza, erosão do solo, congestionamento e tráfego pesado, desmatamentos, entre outros (Borhan et al., 2012).

Os primeiros pesquisadores a modelar a relação entre qualidade ambiental e crescimento econômico foram Grossman \& Krueger (1991), baseando-se nos questionamentos de grupos ambientalistas sobre a liberalização comercial entre os países membros do Tratado Norte-Americano de Livre Comércio (NAFTA), já que alguns poluentes são subprodutos da atividade econômica e que, dada a expansão desta atividade, a tendência seria o aumento da emissão de poluentes. Os estudos revelaram uma relação linear crescente entre poluentes atmosféricos e crescimento do Produto Interno Bruto (Grossman \& Krueger, 1991). Porém, de acordo com os autores, há uma relação em forma de "U" invertido entre poluição ambiental e PIB per capita, a qual foi denominada de "Curva Ambiental de Kuznets". Atualmente, Raftery et al (2017) elaboraram modelos para as maiores economias mundiais, relacionando PIB per capita com emissões de $\mathrm{CO}_{2}$, e elaboraram previsões para 2100 .

No Brasil, alguns estudos utilizando indicadores econômicos e ambientais buscaram verificar a interação entre aqueles fatores. Estudando a expansão econômica, Flores (2017) verificou que houve piora no meio ambiente brasileiro e, ainda, que acordos internacionais -como o Protocolo de Kyoto - e leis ambientais não têm sido suficientes para regular o crescimento econômico orientado a um desempenho ecologicamente sustentável. Anteriormente, Da Silva Collusso et al. (2015) haviam apontado que, nos últimos anos, houve uma relação linear positiva crescente entre o PIB per capita e o desmatamento no Cerrado (savana) do Brasil. Morel et al. (2016) constataram que a relação entre resíduos sólidos e o PIB para o Brasil é não linear, ou seja, os aumentos no Produto Interno Bruto levaram a aumentos nos resíduos sólidos per capita até um ponto de inflexão, no qual o sinal do efeito se inverte e o resíduos sólidos começam a diminuir. Enquanto Dos Santos et al. (2017), numa investigação que vai de 1950 a 2012, verificaram uma relação linear positiva entre PIB per capita e emissões de $\mathrm{CO}_{2}$ no Brasil.

Partindo das considerações já realizadas, avaliar as atividades econômicas no âmbito florestal da produção da madeira nativa e plantada, o seu modelo de crescimento e seu impacto ambiental, permitiria diagnosticar e promover uma economia que adote boas práticas socioambientais no desenvolvimento rural, que garantam o bem-estar da sociedade e o equilíbrio entre produção e conservação.

\section{MATERIAL E MÉTODOS}

O procedimento metodológico inicial foi a construção do referencial teórico sobre o desenvolvimento econômico e da atividade agroflorestal, 
utilizando pesquisa de caráter exploratório. O segundo passo foi a organização da base de dados secundários. Para verificar a influência ou impacto da exploração de produtos madeireiros de florestas nativas e plantadas no desenvolvimento socioeconômico e ambiental, optouse pela utilização de modelagem econométrica com dados longitudinais entre 2000 e 2014 .

Os dados relativos ao Mato Grosso do Sul, para o período de 2000 a 2014, foram obtidos em fontes secundárias e oficiais, disponibilizados nos sites do Instituto Brasileiro de Geografia e Estatística, extraídos por meio do sistema de dados agregados do Sistema IBGE de Recuperação Automática, oriundos do Sistema de Contas Nacionais e da Pesquisa de Produção da Extração Vegetal e Silvicultura (IBGE, 2017); do Sistema de Estimativas de Emissões de Gases de Efeito Estuda (SEEG, 2017); da Secretaria do Estado de Meio Ambiente e Desenvolvimento Econômico (SEMADE, 2017); e do Ministério do Desenvolvimento, Indústria e Comércio Exterior e Serviços (MDIC, 2017). As análises estatísticas foram realizadas por meio do software estatístico $R^{6}$, com as variáveis apresentadas na Tabela 1 .

Os indicadores de atividade econômica e os valores brutos da produção de madeireiros utilizados na pesquisa foram corrigidos aos valores de 2014 através do Índice de Preços ao Consumidor Amplo (IPCA), que os adequou à inflação oficial no período. Naquele momento, de janeiro de 2000 a dezembro de 2014, segundo o IBGE (2017), a inflação foi de 143,18\%.

Três modelos foram utilizados, levando em consideração o PIB e o PIB per capita como variável dependente para analisar o impacto no desenvolvimento econômico, e o $\mathrm{CO}_{2}$ como variável dependente para avaliar o impacto ambiental, a pesquisa em caráter inédito inclui a variável de Grau de Abertura Comercial e seu impacto com a entrada de grandes empresas estrangeiras e seus respectivos investimentos.

A estimação busca a relação linear entre as variáveis dependentes e explicativas, e o método de estimação utilizado foi o Mínimos Quadrados Ordinários (MQO), que otimiza e procura encontrar o melhor ajuste para um conjunto de dados tentando minimizar a soma dos quadrados das diferenças entre o valor estimado e os dados observados. A modelagem utilizou o pacote BETS ${ }^{7}$ do $R$, que inclui os testes de Estacionariedade, Autocorrelação a análise dos resíduos padronizados e o teste de Llung-Box (Ljungand Box, 1978).

\section{RESULTADOS E DISCUSSÃO}

Os dados mostram um crescimento exponencial na atividade madeireira no Mato Grosso do Sul a partir de 2007, onde revelou expansão do setor no estado,

Tabela 1 - Variáveis utilizadas para a estimação dos modelos econométricos

\begin{tabular}{clc}
\hline Nomenclatura & \multicolumn{1}{c}{ Variável } & Unidade \\
\hline PIB & Produto Interno Bruto & $\mathrm{R} \$$ \\
PIBcap & Produto Interno Bruto per capita & $\mathrm{R} \$$ \\
Sil & Produção Bruta Madeireira de Florestas Plantadas & $\mathrm{R} \$$ \\
ProdF1 & Produção Bruta Madeireira de Florestas* & $\mathrm{R} \$$ \\
EmCO 2 & Emissão de CO $\mathrm{C}_{2}$ equivalente da Agropecuária & Tonelada \\
Ext & Produção Bruta Madeireira de Florestas Nativas & $\mathrm{R} \$$ \\
$\mathrm{GA}$ & Grau de Abertura Comercial** & Índice \\
\hline
\end{tabular}

* Para o cálculo da ProdF1, utilizaram-se os somatórios totais anuais do valor bruto de produção dos produtos da floresta nativa e da floresta plantada, oriundos do carvão vegetal, da lenha, da madeira em tora para papel e celulose e da madeira em tora para outras finalidades.

**Para o cálculo do GA, foi utilizada a seguinte fórmula: $\mathrm{GA}^{\mathrm{t}}=\frac{\mathrm{X}^{\mathrm{t}}+\mathrm{M}^{\mathrm{t}}}{\mathrm{PIB}^{\mathrm{t}}}$, em que: GA $\mathrm{G}^{\mathrm{t}}$ o grau de abertura comercial do estado de Mato Grosso do Sul no período t; $\mathrm{X}^{\mathrm{t}}$ é o valor das exportações totais do Estado no período t; $\mathrm{M}^{\mathrm{t}}$ é o valor das importações totais do Estado no período t; e PIB é o Produto Interno Bruto total do Estado no período t.

${ }^{6} \mathrm{R}$ Core Team (2017). R: A language and environment for statistical computing. R Foundation for Statistical Computing, Vienna, Austria. URL https://www.R-project.org/.

${ }^{7}$ Ver: https://bibliotecadigital.fgv.br/dspace/bitstream/handle/10438/18172/Brazilian_Economic_Time_Series_BETS_R_package.pdf 
com variação maior que os demais estados brasileiros, corroborando com os estudos do IBA (2017).

Com relação à utilização do uso da madeira para fins comerciais, observou-se o aumento da extração da madeira originados de reflorestamentos, principalmente para utilização como matéria prima da celulose; enquanto, para as florestas nativas, verificouse uma diminuição do valor bruto da produção a partir de 2004, que tem como principal produto o carvão vegetal, conforme dados extraídos da Pesquisa de Extração Vegetal e Silvicultura (IBGE, 2017), observados na Figura 1.

Um resultado expressivo para o meio ambiente é a variação negativa do uso de florestas nativas no estado, sendo evidências de melhoras no comportamento de sustentabilidade deste setor produtivo.

A expansão e os investimentos no setor atraíram inúmeras indústrias ligadas ao monocultivo de eucalipto para o Estado. A partir de 2009, a junção da "Votorantim Celulose Papel" com a "International Paper" e a "Aracruz Celulose" culminou em um grande complexo industrial: a "Fibria". Naquele ano, este complexo se tornou o maior produtor de celulose do mundo (Kudlavicz, 2011; Perpetua, 2012). Já em 2012, a "Fibria" foi superada pela empresa "Eldorado Brasil", controlada pelo "Grupo JBS" e pela "MCL Empreendimentos", tornando-se a maior fábrica de celulose de fibra curta do mundo (Lopes \& Miranda, 2017).

Verificou-se a diminuição de produtos da madeira originadas das florestas nativas (Figura 1), na qual a exploração é desenvolvida por meio de licenciamentos ambientais, os quais diminuíram nos anos pesquisados. As atividades de exploração dos recursos florestais e seus usos, como o de conversão para uso alternativo do solo por supressão vegetal, têm sido objeto de ampla fiscalização através de investimentos do governo do MS em sistemas de geo-referenciamento e de imagens de satélite, realizando assim, uma fiscalização mais rígida, notadamente nas áreas de preservação permanente (IMASUL, 2017).

Com relação aos ganhos decorrentes do aumento da atividade produtiva do setor agroflorestal refletidos no aumento do PIB na Figura 1, estimou-se também o GA, que é o nível de liberalização do comércio e a orientação da política comercial de Mato Grosso do
Sul (atratividade das empresas), tanto para o mercado interno quanto para o mercado externo. Seu objetivo foi apontar qual a real participação do setor externo no produto doméstico, ou seja, quão aberta ao mercado externo está a economia do Estado do MS, apontado pela Figura 2.

De forma inédita a pesquisa apresenta a influência da abertura de mercado no Mato Grosso do Sul como proxy para análise do impacto na liberdade econômica no desenvolvimento econômico do estado, do setor agroflorestal e no meio ambiente. Esta variável vai de acordo com os estudos mais recentes do Instituto Mackenzie de Liberdade Econômica Estadual (2017) ${ }^{8}$ e o Fraser Institute (2016) ${ }^{9}$, que mostraram que o Brasil é um dos países com menor liberdade econômica, contudo, Mato Grosso do Sul é o estado com maior abertura comercial em relação aos demais estados brasileiros. Os dados comprovam que a abertura comercial medida vem aumentando ao longo do tempo (Figura 2).

Observa-se que, no período de 2000 a 2014, a diferença do saldo comercial entre importações e exportações foi reduzida, bem como houve um ganho no GA, sendo possível constatar que, nos últimos anos, houve majoração da abertura comercial do Mato Grosso do Sul.

\section{Abordagem econométrica}

Com objetivo de mensurar as externalidades geradas pelo setor agroflorestal no crescimento econômico, os modelos econométricos Econômicos (Modelo $1 \mathrm{e}$ 2) e Ambiental (Modelo 3) são:

$$
\begin{gathered}
\text { Modelo 1: } \\
\begin{aligned}
\operatorname{PIB}_{\mathrm{t}}=\beta_{0}+\beta_{1} \text { silv }_{\mathrm{t}}+\beta_{2} \text { ext }_{\mathrm{t}}+\beta_{3} \mathrm{ga}_{\mathrm{t}}+\varepsilon_{\mathrm{t}} \\
\text { Modelo 2: }
\end{aligned} \\
\text { PIBpc }_{\mathrm{t}}=\beta_{0}+\beta_{1} \text { silv }_{\mathrm{t}}+\beta_{2} \text { ext }_{\mathrm{t}}+\beta_{3} \mathrm{ga}_{\mathrm{t}}+\varepsilon_{\mathrm{t}} \\
\text { Modelo 3: } \\
\mathrm{CO}_{2 \mathrm{t}}=\beta_{0}+\beta_{1} \text { silv }_{\mathrm{t}}+\beta_{2} \text { ext }_{\mathrm{t}}+\beta_{3} \mathrm{ga}_{\mathrm{t}}+\varepsilon_{\mathrm{t}}
\end{gathered}
$$

O modelo 1 e modelo 2 foram estimados para analisar o impacto da produção madeireira de silvicultura e extrativismo, além do grau de abertura comercial no desenvolvimento econômico medido pelo PIB no modelo 1 e PIB per capita no modelo 2.

\footnotetext{
${ }^{8} \mathrm{http}$ //liberdadeeconomica.mackenzie.br/artigos/arquivo/artigo/indice-mackenzie-de-liberdade-economica-estadual/

${ }^{9} \mathrm{http}$ ///iberdadeeconomica.mackenzie.br/artigos/arquivo/artigo/indice-mackenzie-de-liberdade-economica-estadual/
} 


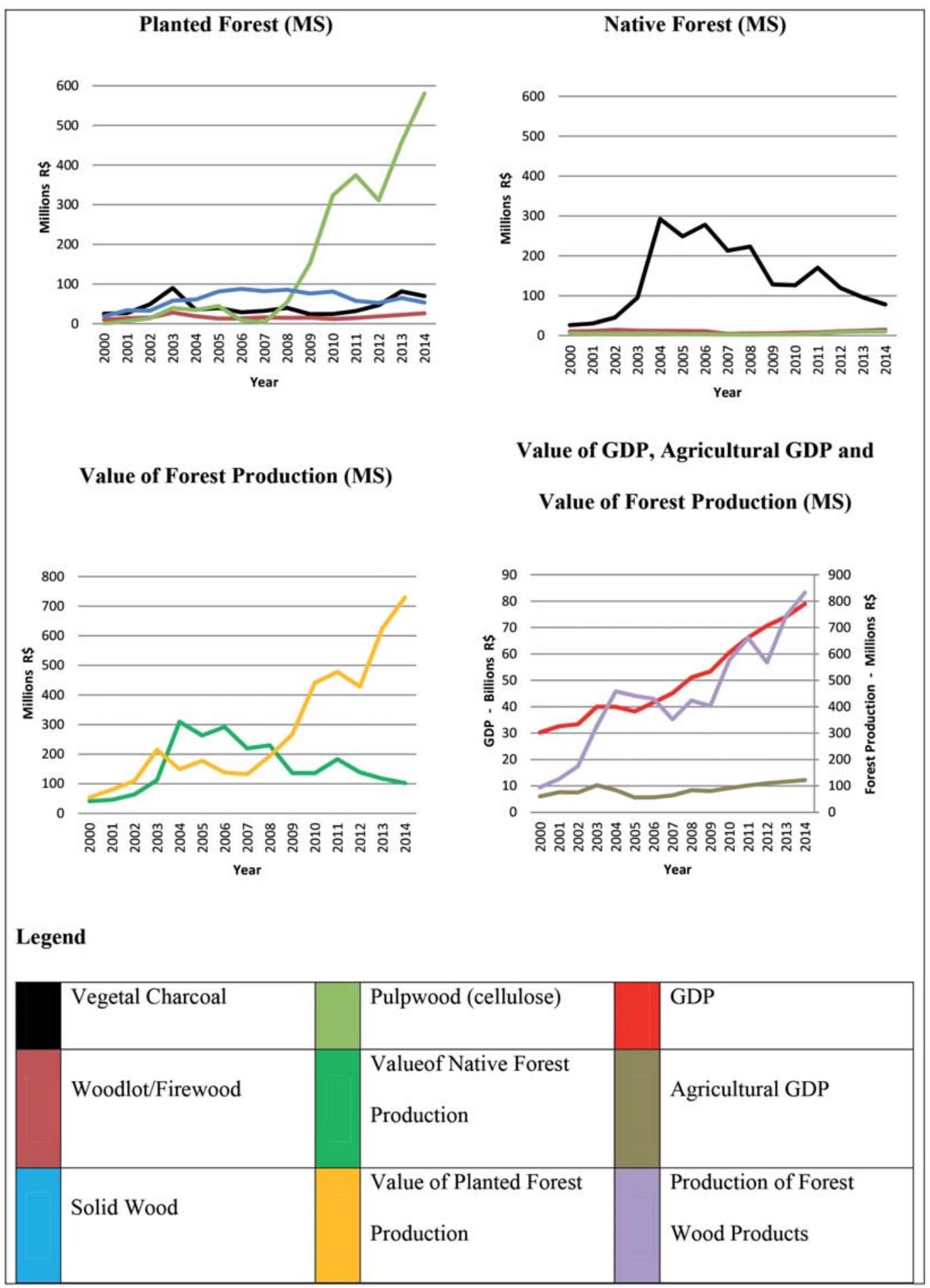

Figura 1 - Evolução da produção dos produtos madeireiros, do PIB, e do PIB Agro no Estado de Mato Grosso do Sul.

Fonte: IBGE, 2017. 


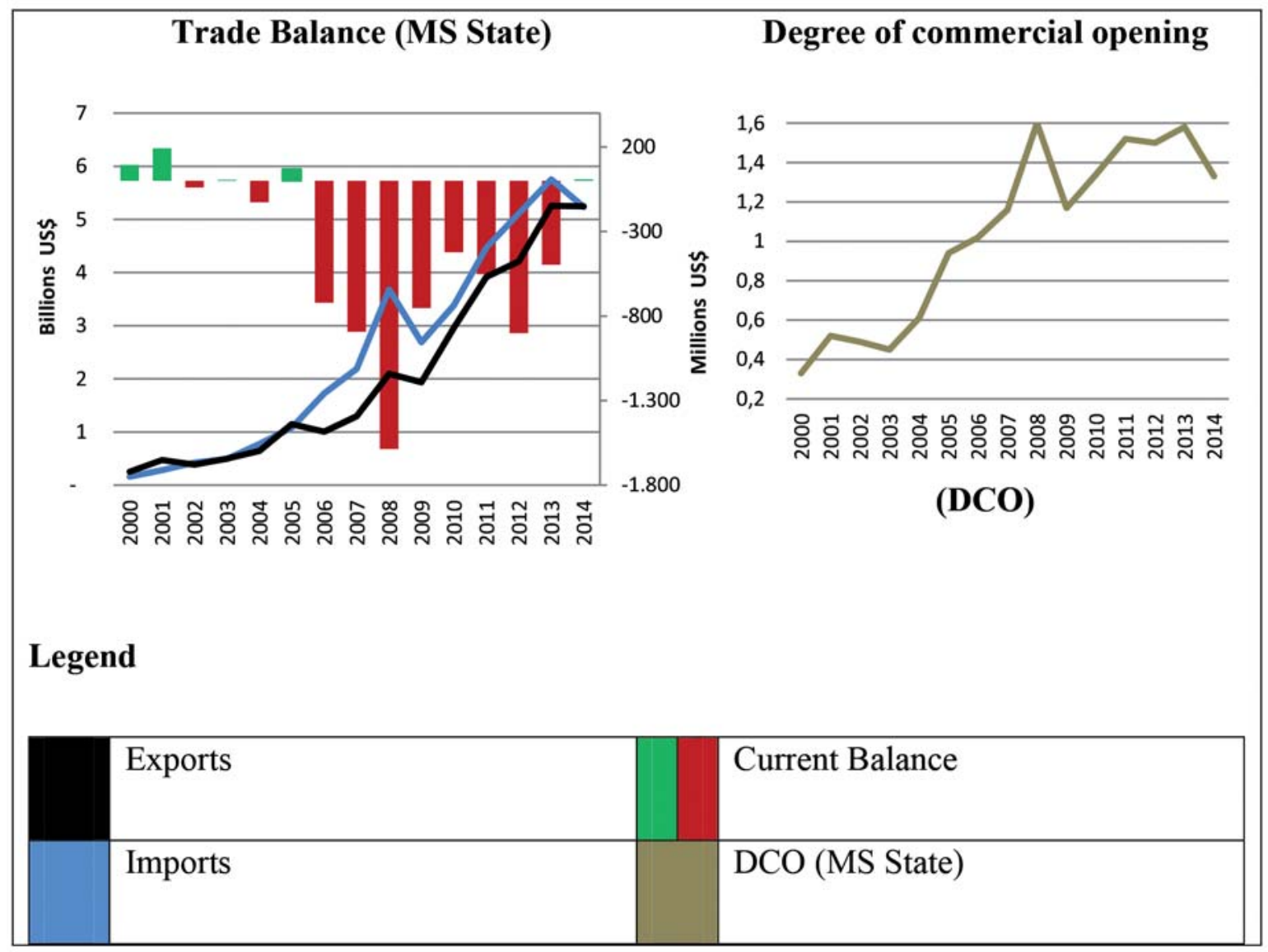

Figura 2 - Evolução da balança comercial e do grau de abertura comercial no Estado de Mato Grosso do Sul, no período de 2000 a 2014.

Fonte: MDIC, 2017; SEMADE, 2017.

Para o modelo ambiental foi estimado como variável dependente as emissões de $\mathrm{CO}_{2}$ para o estado de Mato Grosso do Sul, e as variáveis explicativas foram as mesmas dos modelos $1 \mathrm{e} 2$, como constructo comparativo da análise.

Para o modelo 1, a produção de madeiras plantadas estimaram um impacto positivo e significante no PIB de Mato Grosso do Sul, a produção de extrativismo foi positiva porém, insignificante estatisticamente. A abertura comercial, ou maior liberdade econômica leva há um impacto positivo e significativo para a economia, demonstrando importante componente para o desenvolvimento do estado.

O modelo 2 apresentou o mesmo resultado no modelo 1, com impactos positivos da produção de madeiras plantadas e abertura comercial para a renda média da população do estado.

Para o meio ambiente (modelo 3 ) os resultados apresentaram evidencias empíricas de nenhuma influência da produção de madeira plantada nas emissões de $\mathrm{CO}_{2}$, pois, o coeficiente de variação não foi estatisticamente significante. A produção madeireira de madeiras nativas apresentaram impacto positivo nas emissões de $\mathrm{CO}_{2}$, importante resultado, evidenciando que além de ser negativo o uso de madeiras nativas, sua produção aumenta o impacto ambiental, com o uso de manejo da terra para o extrativismo. Porém, a liberdade econômica, caracterizada pela abertura comercial do estado, apresentou impacto negativo nas emissões de $\mathrm{CO}_{2}$, revelando evidências que a maior abertura comercial, 
Tabela 2 - Resultados das equações dos modelos estimados

\begin{tabular}{|c|c|c|c|}
\hline & \multicolumn{3}{|c|}{ Dependent variable: } \\
\hline & pib & pibpc & $\operatorname{co} 2$ \\
\hline & (1) & $(2)$ & (3) \\
\hline silv & $\begin{array}{c}0.00002 * * * \\
(0.00000)\end{array}$ & $\begin{array}{c}0.00001 \cdots \\
(0.00000)\end{array}$ & $\begin{array}{c}0.00000 \\
(0.00000)\end{array}$ \\
\hline ext & $\begin{array}{c}0.00001 \\
(0.00001)\end{array}$ & $\begin{array}{l}-0.00000 \\
(0.00000)\end{array}$ & $\begin{array}{r}0.00001^{\cdots *} \\
(0.00000)\end{array}$ \\
\hline ga & $\begin{array}{c}0.546^{* *} \\
(0.131)\end{array}$ & $\begin{array}{c}0.250^{* *} \\
(0.070)\end{array}$ & $\begin{array}{c}-0.124^{\cdots} \\
(0.031)\end{array}$ \\
\hline Constant & $\begin{array}{c}13.919^{* *} \\
(0.087)\end{array}$ & $\begin{array}{c}2.623^{\cdots *} \\
(0.047)\end{array}$ & $\begin{array}{c}8.234^{* *} \\
(0.020)\end{array}$ \\
\hline Observations & 15 & 15 & 15 \\
\hline & 0.965 & 0.942 & 0.711 \\
\hline Adjusted $\mathrm{R}^{2}$ & 0.955 & 0.926 & 0.632 \\
\hline Residual Std. Error $(\mathrm{df}=11)$ & 0.123 & 0.066 & 0.029 \\
\hline F Statistic $(\mathrm{df}=3 ; 11)$ & $99.808^{* \cdots}$ & $59.285^{* * *}$ & $9.010^{* * *}$ \\
\hline
\end{tabular}

diminui o impacto ambiental e promove desenvolvimento sustentável.

Os resultados são antagônicos com pesquisas de (Pereira, 2012; Martins, 2013) e (Ortigoza \& Senna, 2016). E semelhante com os resultados do (IEA, 2017).

\section{CONSIDERAÇÕES FINAIS}

A abertura comercial (liberdade econômica) e a política de investimentos baseada em financiamentos públicos e privados para o setor de florestas plantadas favoreceu o desenvolvimento econômico do Estado de Mato Grosso do Sul. Os resultados econométricos mostraram que com aumento da produção madeireira de florestas plantadas houve ganhos ambientais efetivos, que ocorreram com a diminuição de $\mathrm{CO}_{2}$ equivalente, emitidos pela atividade agropecuária, evidenciando aspectos tecnológicos de economia limpa.

Há evidências empíricas que a Economia Verde ou Bioeconomia em Mato Grosso do Sul foi favorecida pelo maior Grau de Abertura Comercial, que impactaram de forma positiva a política de atração de investimentos para o setor agroflorestal no Estado, promovendo significativa participação no desenvolvimento do estado via setor de silvicultura.

Os modelos e análises realizadas mostraram-se eficazes para evidenciar os efeitos das variáveis analisadas no aumento/redução do PIB e, consequentemente, no desenvolvimento rural e a busca pela economia verde, e desta forma, podem auxiliar nas tomadas de decisões dos policy-makers e produtores, com reflexos desejáveis na balança comercial.

São poucos trabalhos relacionando o setor agroflorestal, liberdade econômica e impacto econômico e ambiental, nesse contexto, a presente pesquisa abre caminho para incentivar novas abordagens, novos modelos e a continuidade de uma análise robusta e empírica sobre o desenvolvimento baseado em sustentabilidade.

\section{BIBLIOGRAFIA}

ANDRADE, D. C.; ROMEIRO, A. R. Degradação ambiental e teoria econômica: algumas reflexões sobre a "economia dos ecossistemas". Revista Economia, Brasília, v. 12, n. 1, p. 3-26, jan/abr de 2011 . 
BORHAN, H.; AHMED, E. M.; HITAM, M. The impact of $\mathrm{CO}_{2}$ on economic growth in ASEAN 8. Procedia - Social and Behavioral Sciences, v. 35, p. 389-397, 2012.

DALCHIAVON, E. C.; DE MELLO, G. R. Produção do setor rural e o desenvolvimento socioeconômico: um estudo nos municípios paranaenses. Revista da FAE, v. 18, n. 1, p. 86-99, 2015.

DA SILVA COLUSSO, M. V.; PARRÉ, J. L.; ALMEIDA, E. S. Degradação Ambiental e Crescimento Econômico: A Curva De Kuznets Ambiental para o Cerrado. Revista de Economia e Agronegócio - REA, v. 10, n. 3, 2015 .

DOS SANTOS, R. P.; RIBEIRO, A. R.; DE MENDONCA, K. V. Crescimento Econômico e Degradação da Qualidade do Ar: O Caso Brasileiro. Encontros Universitários da UFC, v. 1, p. $2680,2017$.

FÁvero, L. P. et al. Análise de dados: modelagem multivariada para tomada de decisões. Rio de Janeiro: Elsevier, 2009.

FAO/IPCC. Expert meeting on land use, climate change and food security, Rome, Italy, 23-25 January 2017. Disponível em: http:// www.ipcc.ch/pdf/supporting-material/ EM_FAO_IPCC_report.pdf. Acesso em: abril de $201 \overline{7}$.

FLORES, R. J. G. Teste da hipótese da curva de Kuznets ambiental para os países do BRICS. 2017. 143 f. Dissertação (Mestrado em Economia). Universidade Federal de Uberlândia, Uberlândia, 2017.

GROSSMAN, G. M.; KRUEGER, A. B.

Environmental impacts of a North American free trade agreement. USA: National Bureau of Economic Research, NBER Working Paper no 3914, nov. 1991.

HERRERA, G. P.; LOURIVAL, R.; COSTA, R. B.; MENDES, D. R. F.; MOREIRA, T. B. S.; ABREU, U. G. P.; CONSTANTINO, M. Econometric analysis of income, productivity and diversification among smallholders in Brazil, Land Use Policy, Volume 76, 2018. Pages 455-459, https://doi.org/10.1016/j.landusepol.2018.02.025.
IBGE. Instituto Brasileiro de Geografia e Estatística. Disponível em: www.ibge.gov.br/ sidra. Acesso em: abril de 2017.

IEA. Instituto de Economia Agrícola. Banco de dados. São Paulo: IEA. Disponível em: http://www.iea.sp.gov.br/out/floresta/ mercados2.php. Acesso em: abril de 2017.

IMASUL. Instituto do Meio Ambiente de Mato Grosso do Sul. Disponível em: Resoluções. http://www.imasul.ms.gov.br/wp-content/uploads/ sites/74/2015/06/Manual-2015.pdf. Acesso em: abril de 2017.

KUDLAVICZ, M. Dinâmica agrária e a territorialização do complexo celulose/ papel na microrregião de Três Lagoas/MS. 2011.177 f. Dissertação (Mestrado em Geografia). Universidade Federal de Mato Grosso do Sul, Três Lagoas, 2011.

LELIS, L. R. M.; AVELINO JÚNIOR, F. J.Territorialização do Complexo EucaliptoCelulose-Papel e Resistência Camponesa Em Três Lagoas-MS. Caminhos de Geografia, v. 17, n. 58, p. 81-102, 2016.

LOPES, C. R.; MIRANDA, N. Desenvolvimento econômico de Três Lagoas/MS, desertos verdes e os cativeiros de papel. Revista de Estudos Jurídicos UNESP, Franca, ano19, n.30, p.118, ago-dez, 2015.

MARTINS, S. R. O. Trabalho escravo: sujeição ou escravidão? Multitemas, n. 3, 2016.

MDIC. Ministério do Desenvolvimento, Indústria e Comércio Exterior. Balança Comercial: Unidades da Federação. Disponível em: http://www.mdic.gov.br/comercio-exterior/ estatisticas-de-comercio-exterior/balancacomercial-brasileira-unidades-da-federacao. Acesso em: abril de 2017.

MOREL, B. L. G.; TRIACA, L. M.; DE SOUZA, O. T. Desenvolvimento econômico e a disposição de resíduos sólidos no Brasil: um teste da hipótese da Curva Ambiental de Kuznets (CKA) para os municípios brasileiros. Revista ESPACIOS, v. 37 , n. 17 , p. $22,2016$. 
ORTIGOZA, F. S. S.; SENNA, R. J. Caracterização do segmento de florestas plantadas de eucalipto em Mato Grosso do Sul. Revista de Política Agrícola, v. 25, n. 2, p. 74-89, 2016.

PEREIRA, A. Precarização e (des) estruturação do trabalho nas carvoarias. Revista Pegada, v. 8, n. 2, 2012.

PERPETUA, G.M. A mobilidade espacial do capital e da força de trabalho na produção de celulose e papel: um estudo a partir de Três Lagoas (MS). 251p. Dissertação (Mestrado em Geografia). Universidade Federal da Grande Dourados, Dourados, 2012.
RAFTERY, A. E.; ZIMMER, A.; FRIERSON, D. M. W.; STARTZ, R.; LIU, P. Lessthan $2 æ \% \mathrm{C}$ warmingby 2100 unlikely. NATURE CLIMATE CHANGE, Springer Nature, 2017. https:// www.nature.com/articles/nclimate3352

SEEG. Sistema de Estimativa de Emissões de Gases de Efeito Estufa. Observatório do clima. Disponível em: http://

plataforma.seeg.eco.br/sectors/agropecuaria. Acesso em: abril de 2017.

SEMADE. Secretaria do Estado de Meio Ambiente e Desenvolvimento

Econômico. Contas Regionais. Disponível em: http:/www.semade.ms.gov.br/wp-content/uploads/ sites/20/2016/12/PIB-Municipal-2010-2014-1.pdf. Acesso em: abril de 2017.

Recebido para publicação em 17/3/2018 e aprovado em 11/12/2018. 